\title{
Efficient Red-Shifted Dispersive Wave in a Photonic Crystal Fiber for Widely Tunable Mid-Infrared Wavelength Generation
}

Jinhui Yuan

Beijing University of Posts and Telecommunications

Xinzhu Sang

Beijing University of Posts and Telecommunications

Qiang wu

Technological University Dublin, qiang.wu@tudublin.ie

See next page for additional authors

Follow this and additional works at: https://arrow.tudublin.ie/engscheceart

\section{Recommended Citation}

Yuan, J., Sang, X., Wu, Q., Yu, C. Farrell, G. and Hou, L. Efficient red-shifted dispersive wave in a photonic crystal fiber for widely tunable mid-infrared wavelength generation. Laser Physics Letters, 10 (2013) 045405 (5pp) doi;10.1088/1612-2011/10/4/045405

This Article is brought to you for free and open access by the School of Electrical and Electronic Engineering at ARROW@TU Dublin. It has been accepted for inclusion in Articles by an authorized administrator of ARROW@TU Dublin. For more information, please contact arrow.admin@tudublin.ie, aisling.coyne@tudublin.ie, gerard.connolly@tudublin.ie.

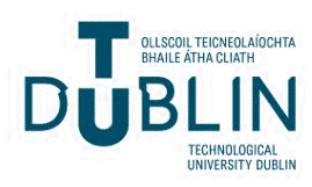




\section{Authors}

Jinhui Yuan, Xinzhu Sang, Qiang wu, Chongxiu Yu, K.R. Wang, B.B. Yan, X.W. Shen, Y. Han, G.Y. Zhou, Yuliya Semenova, Gerald Farrell, and L.T. How 


\title{
Efficient red-shifted dispersive wave in a photonic
}

\section{crystal fiber for widely tunable mid-infrared wavelength}

\author{
generation

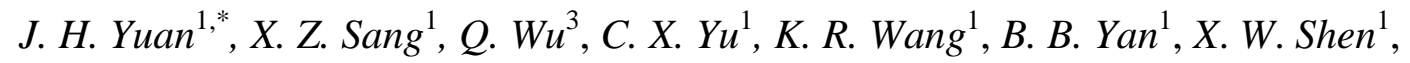 \\ Y. $\mathrm{Han}^{2}$, G. Y. Zhou ${ }^{2}$, Y. Semenova ${ }^{3}$, G. Farrell ${ }^{3}$, and L. T. Hou ${ }^{2}$ \\ ${ }^{1}$ State Key Laboratory of Information Photonics and Optical Communications (Beijing University \\ of Posts and Telecommunications), P.O. Box163 (BUPT), 100876 Beijing, China \\ ${ }^{2}$ Institute of Infrared Optical Fibers and Sensors, Physics Department, Yanshan University, \\ 066004 Qinhuangdao, China \\ ${ }^{3}$ Photonics Research Center, School of Electronic and Communications Engineering, \\ Dublin Institute of Technology, Kevin Street, Dublin 8, Ireland \\ *Email: yuanjinhui81@163.com
}

Abstract: Red-shifted dispersive waves (DWs) at the mid-infrared wavelengths are generated for the first time by pumping a photonic crystal fiber (PCF) with two zero dispersion wavelengths specially designed and fabricated in our lab, and the central wavelength of the soliton generated lies on the negative slope of the PCF dispersion profile. The experimental result shows that the conversion efficiency $\eta$ of red-shifted DWs increases from 5\% to $15 \%$ as the input power changes from 100 to $300 \mathrm{~mW}$ with the pump wavelength of $810 \mathrm{~nm}$. By adjusting both the pump wavelength and power, the tunable range of the wavelength generated by red-shifted DWs can be over $100 \mathrm{~nm}$.

Key words: photonic crystal fiber (PCF), red-shifted dispersive waves (DWs), mid-infrared wavelengths

\section{Introduction}

Supercontinuum generation [1] for the broadband light source in photonic crystal fibers (PCFs) [2,3] is an interesting nonlinear phenomenon. The generation mechanism for the short pulses is mainly based on the notions of high-order soliton fission [4]. However, some interesting nonlinear applications have involved the generation of narrowband frequency components. The unique flexibility of the dispersion and nonlinearity management provided by PCFs is a key enabling mechanism for the generation of narrowband light source within a wide wavelength range [5,6]. The PCF-based nonlinear frequency conversion techniques have been developed to efficiently convert the pump power into the targeted spectral band. In addition to utilizing the four-wave mixing (FWM) excited near the zero dispersion wavelength [7-12], another well-known technique is based on the generation of dispersive waves (DWs) mediated by the fiber solitons.

The DWs can be generated and enhanced on the short- or long-wavelength side of the pump wavelength when the resonance condition involving the high-order dispersion terms is satisfied in a process of soliton self-frequency shift (SSFS) [13]. Cristiani et al. firstly studied the generation mechanism of DWs in PCFs [14]. Chang 
et al and us achieved the efficient blue-shifted DWs at the visible wavelength $[15,16]$. The red-shifted DWs were only demonstrated at a shorter wavelength than the mid-infrared wavelength [17-20], and they couldn't be used as the short pulse source because of the low conversion efficiency and narrow wavelength-tunable range. In this letter, the DWs are generated at the mid-infrared wavelength in a silica PCF. The conversion efficiency $\eta$ of $15 \%$ and wavelength-tunable range of over $100 \mathrm{~nm}$ are experimentally achieved. To the best of our knowledge, this is the first report on the efficient generation of red-shifted DWs at the mid-infrared wavelength. This significant radiation is particularly useful for the generation of mid-infrared ultrashort pulse source for the extensive applications in the biophotonics, spectroscopy, and the ultrafast science and technology.

\section{The PCF properties and experiment}

Fig. 1 (a) shows the cross-section of the silica PCF specially designed and fabricated by the improved stack-and-draw technology in our lab, where the core diameter is 2.1 $\mu \mathrm{m}$ and the relative size of hole is 0.85 . The silica material prepared by the plasma chemical vapour deposition (PCVD) is purified prior to the fiber drawing by the complexing method of rectification and adsorption to greatly reduce the absorption losses of PCF at the mid-infrared wavelengths induced by the chlorides, chlorine-hydrogen compounds, $\mathrm{OH}$, metal ions, and metallic and non-metallic oxides [21]. Fig. 1 (b) shows the calculated group velocity dispersion and effective mode area for the fundamental mode, where the two zero dispersion wavelengths are 755 $\mathrm{nm}$ and $1325 \mathrm{~nm}$, respectively. The existence of two zero dispersion wavelengths makes it possible to generate the DW radiation on the short- or long-wavelength sides of an appropriately selected pump wavelength because the resonant radiation is always created across the zero-dispersion wavelength. The value of the effective mode area increases from 3.1 to $3.55 \mu \mathrm{m}^{2}$ as the wavelength shifts from 780 to 1265 $\mathrm{nm}$, which indicates that the effective mode area has a determinate effect on the process of SSFS.

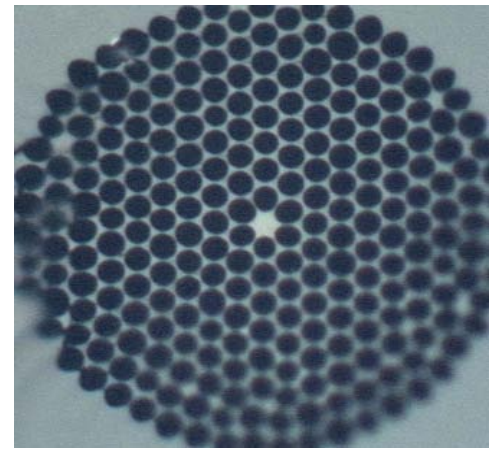

(a)

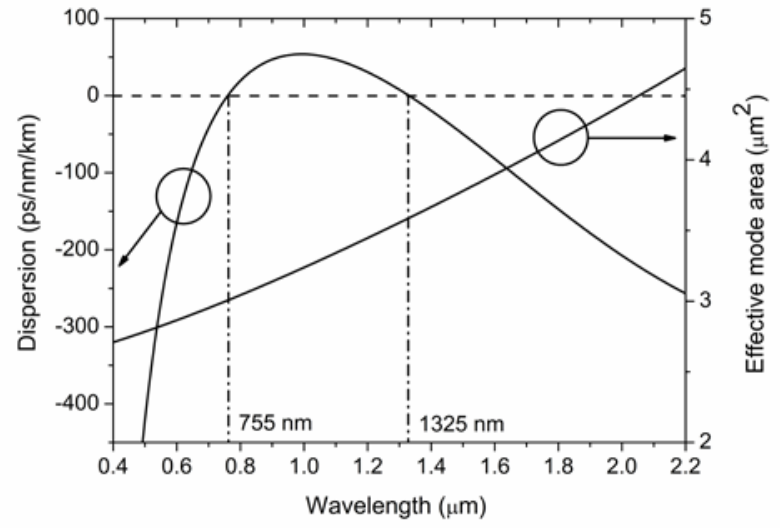

(b)

Fig.1. (a) The cross-section of PCF used in the experiment. (b) Group-velocity dispersion and effective mode area calculated for the fundamental mode, the vertical dashed-lines indicating to the zero dispersion wavelengths of $755 \mathrm{~nm}$ and $1325 \mathrm{~nm}$. 
The experiment principle chart is shown in Fig.2. The pump source used in the experiments is a mode-locked Ti: sapphire ultrafast laser with the tunable working wavelength range of 780 to $980 \mathrm{~nm}$ and the full width at half maximum (FWHM) of $120 \mathrm{fs}$ at a pulse repetition rate of $76 \mathrm{MHz}$. The input power is controlled by a variable attenuator, and an isolator is inserted to block the back-reflection from the input tip of the fiber into the laser cavity. The laser pulses are coupled by the $40 \times$ objective lens with the numerical apertures of 0.65 into a section of PCF, and the coupling efficiency can be up to $65 \%$. The CCD1 and CCD2 are used to check the coupling state of input field and observe the output mode field. The fundamental mode can be selectively excited through changing the distance between the input tip of the fiber and the lens to exactly adjust the angle between the input beam and the fiber axis (the offset pumping technique). The optical beam goes through the first split-beam mirror, one part is coupled into the power meter, and the other part is coupled into the PCF span of $45 \mathrm{~cm}$ length. The fiber length is optimized so that the DWs at the mid-infrared wavelength do not experience too much material absorption to keep the generation level of DW sufficiently high. The output spectra of light from the PCF section are respectively monitored by two optical spectrum analyzers (OSA, Avaspec-256 and Avaspec-NIR-256) with the measurement ranges of 200 to $1100 \mathrm{~nm}$ and 900 to $2500 \mathrm{~nm}$ and the wavelength resolutions of $0.025 \mathrm{~nm}$ and $15 \mathrm{~nm}$.

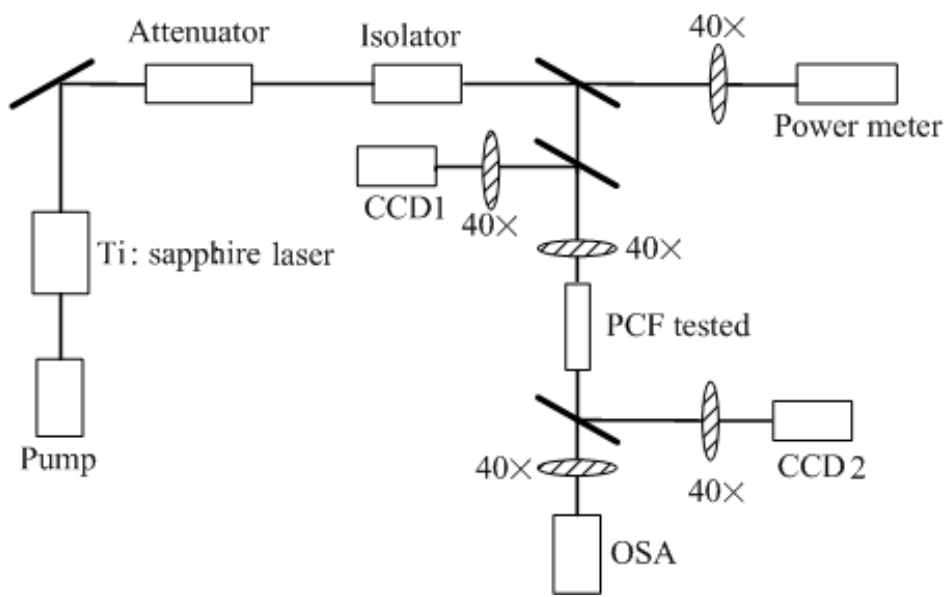

Fig.2 The experimental principle chart.

\section{Results and discussion}

The formation of soliton-like features is experimentally observed in the spectrum of the light output from the PCF for a pump wavelength of $780 \mathrm{~nm}$ and a pump power of $300 \mathrm{~mW}$. Fig. 3 shows the experimental result and also a simulation result calculated using the nonlinear Schrödinger equation (NLSE) [22, 23], taking into account the full complexity of the fiber group velocity dispersion, the Kerr and the Raman contributions to the nonlinearity, the self-steepening (SS), and the loss of pump field due to the coupling between the guided and leaky fiber modes, along with the nonlinear polarization term to include the influence of the wavelength-dependent effective mode area and give a correct definition of the local field intensity. And the case of fourth-order dispersion is considered to exactly predict two frequency-detuned DWs generated at each side of the red-shifted solitons. In the simulation, the detailed 
parameters are as follows: the pump peak power $P_{\mathrm{p}}=36 \mathrm{~kW}$, the FWHM of pulse $T_{\mathrm{FWHM}}=120 \mathrm{fs}$, the nonlinear-index coefficient of fused silica $n_{2}=2.6 \cdot 10^{-20} \mathrm{~m}^{2} / \mathrm{W}$, the dispersion coefficient $\beta_{2}=-1.7366 \mathrm{ps}^{2} / \mathrm{km}, \beta_{3}=0.4575 \cdot 10^{-2} \mathrm{ps}^{3} / \mathrm{km}$, and $\beta_{4}=-1.1878 \cdot 10^{-6}$ $\mathrm{ps}^{4} / \mathrm{km}$ at the pump wavelength of $780 \mathrm{~nm}$, the fractional contribution of the Raman response $f_{\mathrm{R}}=0.18$, the characteristic times of the Raman response $\tau_{1}=12.5 \mathrm{fs}$, and $\tau_{2}=32$ fs. The PCF length is $45 \mathrm{~cm}$, the nonlinear coefficient $\gamma$ is a function of wavelength-dependent effective mode area, and the loss is calculated from extrapolation of the fit following the form $\alpha(\lambda)=A \exp (-\alpha / \lambda)$. As seen from Fig. 3, the experimental and simulation results are in good agreement.

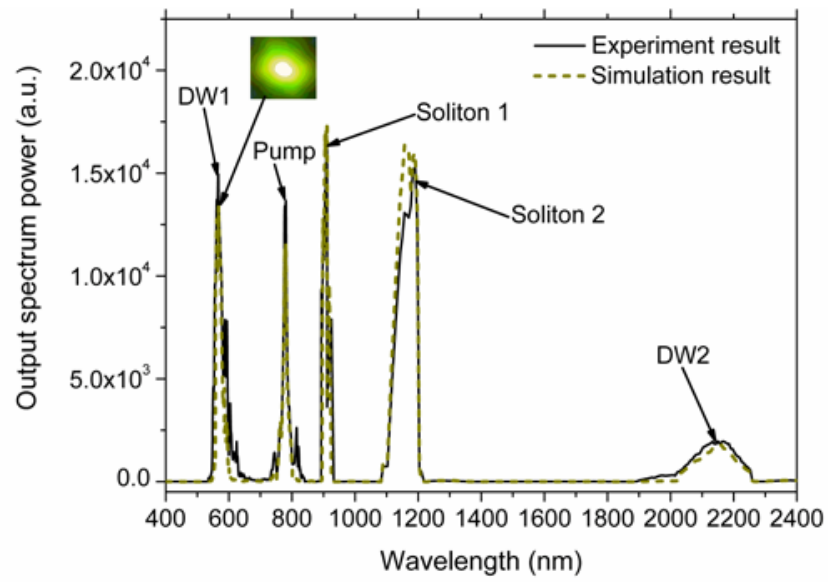

Fig.3. Output spectra for a pump wavelength at $780 \mathrm{~nm}$ and an average power of $300 \mathrm{~mW}$. The short-dotted line corresponds to the simulation result using the NLSE assuming the same pump conditions as the experimental case. The inset shows the observed output far field for a dispersive wave at a visible wavelength of $580 \mathrm{~nm}$ (yellow-green light).

The pump pulse energy is kept to a certain range so that the nonlinear processes are dominated by the formation of the fundamental soliton. At the initial stage of pulse evolution, because the pump pulse lies within the anomalous dispersion region, it tends to evolve toward soliton due to the interplay between the negative dispersion and the self-phase modulation (SPM) as it propagates through the PCF. The fundamental soliton 1 at $910 \mathrm{~nm}$ lies on the positive slope of the fiber dispersion profile ( $\beta_{3}=\partial^{3} \beta / \partial \omega^{3}>0$, where $\beta$ is the relevant mode propagation constant), the higher-order dispersion induces a perturbation in the soliton, and the resonant dispersive wave is generated from the phase matching condition taking into account the nonlinear phase of the soliton. The dispersive wave 1 (DW1) with respect to soliton 1 is predominantly the blue-shifted branch. The experimental results for the DW1 radiation at a wavelength of $566 \mathrm{~nm}$ agrees well with the NLSE simulation results, as shown in Fig.3, where the inset corresponds to the observed far field of DW1, showing that the field intensity reaching the maximum at the center of the fiber core and monotonically decreasing with the distance from the center of the core. Because of a continuous red shift induced by the intra-pulse Raman scattering (IRS), the soliton 2 at $1190 \mathrm{~nm}$ is clearly seen in both experimental and theoretical spectra. Since the central wavelength of soliton 2 is shifted to the region of a negative slope $\left(\beta_{3}<0\right)$, as first predicted by Akhmediev et al [24], it is possible to generate a 
dispersive wave at a wavelength longer than the central wavelength of soliton 2 when the effect of fourth-order dispersion is considered. As presented in Fig.3, the soliton 2 evidently radiate the red-shifted dispersive wave 2 (DW2), observed as a spectral component centered at $2163 \mathrm{~nm}$. Below the properties of soliton 2 and DW2 will be further analyzed.

As shown in Refs [17-20], to conserve the overall energy of the photons, the solitons frequency gets shifted in the spectral direction opposite to that of the DWs. Thus, when the pump operates at the wavelength of $810 \mathrm{~nm}$ and the average power increases from 100 to $300 \mathrm{~mW}$, the central wavelength of soliton $2 \lambda_{\mathrm{s}}$ shifts from 1185 to $1265 \mathrm{~nm}$, and the central wavelength of generated DW2 $\lambda_{\mathrm{DW}}$ is in the range of 2165 to $2105 \mathrm{~nm}$, as shown in Fig.4 (a) and (b). The conversion efficiency $\eta$ can be defined as the ratio of the output power $P_{\mathrm{s}}$ of soliton 2 and $P_{\mathrm{DW} 2}$ of DW2 and the incident power $P_{\mathrm{p} 0}$ of pump. When the pump power changes from 100 to $300 \mathrm{~mW}$, for the coupling efficiency of $65 \%$, the $P_{\mathrm{s}}$ are measured to be $18.2 \mathrm{~mW}, 29.9 \mathrm{~mW}$, and 33.15 $\mathrm{mW}$, and the corresponding $P_{\mathrm{DW} 2}$ are $3.25 \mathrm{~mW}, 14.3 \mathrm{~mW}$, and $29.25 \mathrm{~mW}$. As seen from Fig.4 (c), $\eta$ of soliton 2 reduces from 28\% to 17\% while $\eta$ of DW2 increases from $5 \%$ to $15 \%$. Additionally when the pump power is higher than $300 \mathrm{~mW}, \lambda_{\mathrm{s}}$ remains nearly constant within a wide range of input power. The cancellation of SSFS accompanied by the remarkable amplification of DW2 is considered to be due to the spectral recoil from DW2, which acts on the soliton 2 and compensates for the Raman frequency shift.

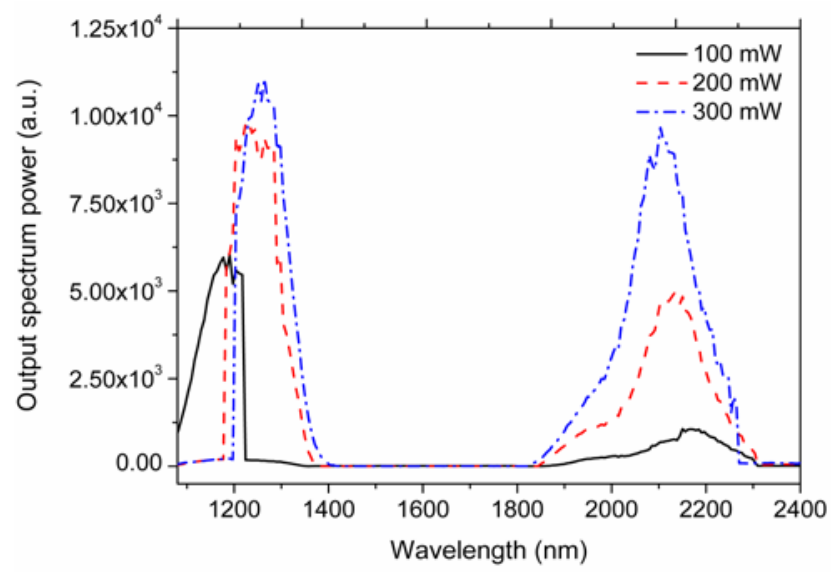

(a)

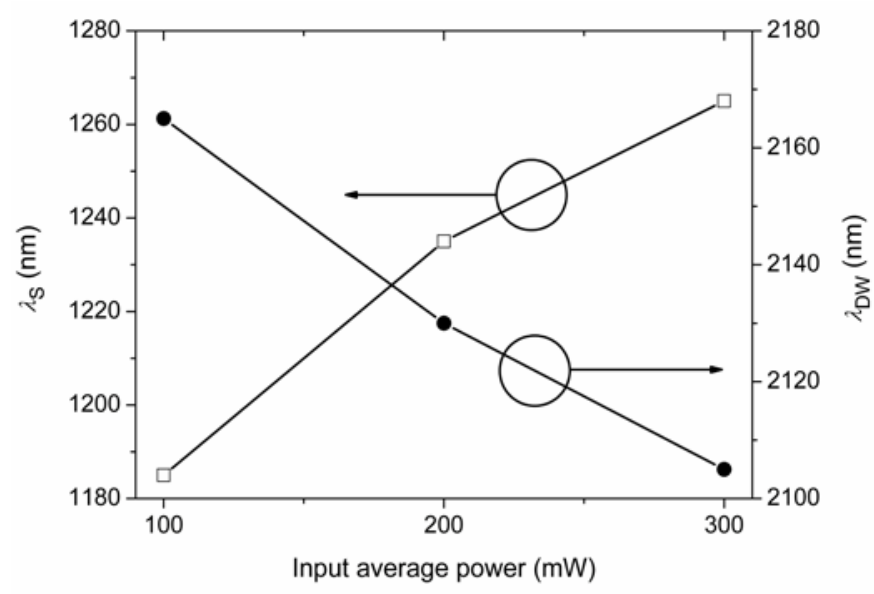


(b)

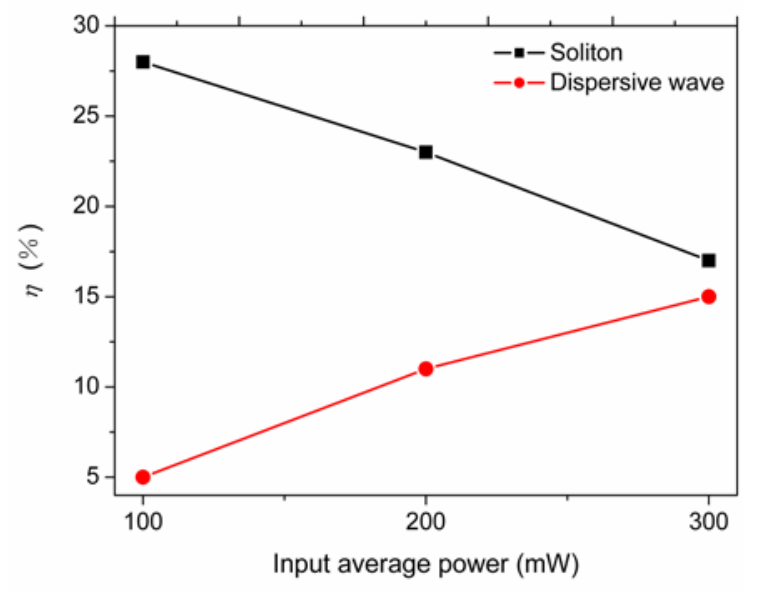

(c)

Fig.4 (a) Output spectra of soliton 2 and DW2 when the pump operates at $810 \mathrm{~nm}$ and the average power increases from 100 to $300 \mathrm{~mW}$. (b) and (c) show $\lambda_{\mathrm{s}}, \lambda_{\mathrm{DW}}$ and $\eta$ of soliton 2 and DW2 as functions of input average power.

By changing both the pump power and the pump wavelength, the DW2 can be efficiently generated over a broad wavelength range. This is due to the special dispersion and nonlinear characteristics of the PCF. As presented in Fig.5, when the input power changes from 100 to $300 \mathrm{~mW}$ with pumping wavelengths of $780 \mathrm{~nm}, 790$ $\mathrm{nm}, 800 \mathrm{~nm}$, and $810 \mathrm{~nm}$, the DW2 radiation can be generated in the wavelength range of 2208 to $2162 \mathrm{~nm}, 2191$ to $2141 \mathrm{~nm}, 2177$ to $2122 \mathrm{~nm}$, and 2165 to $2105 \mathrm{~nm}$, respectively, and the tunable range of $\lambda_{\mathrm{DW}}$ is over $100 \mathrm{~nm}$.

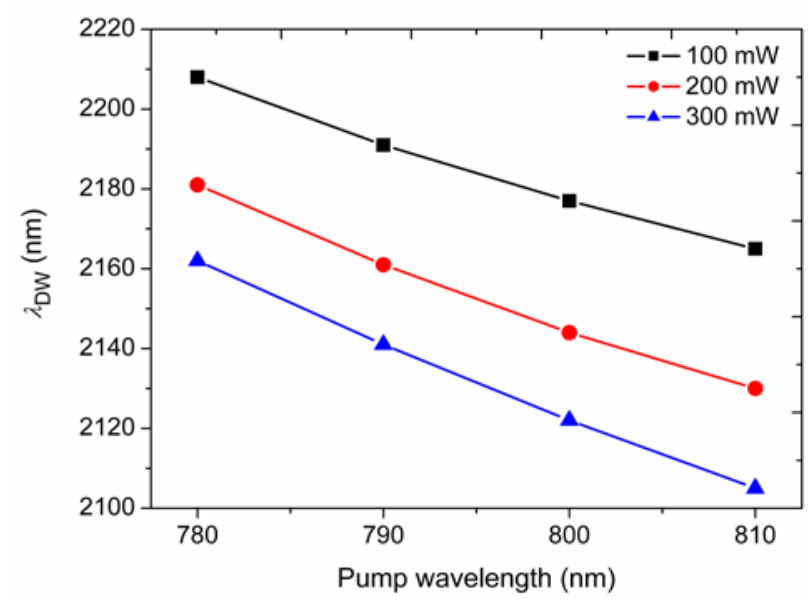

Fig.5 The relationship between $\lambda_{\mathrm{DW}}$ and the pump wavelength.

The widths of short pulses generated by the DW2 can be measured by the Talbot effect. The short pulses pass the grating, and reach the detector which is away from $n$ time Talbot-distance after the grating. The ratio of diffraction intensity $S(T)$ between the transparent and no-transparent part is measured, and the pulse temporal width $T$ can be calculated by the relation curve between $S(T)$ and $T$. Because of the normal dispersion and the SPM, when pumping at $780 \mathrm{~nm}, 790 \mathrm{~nm}, 800 \mathrm{~nm}$, and $810 \mathrm{~nm}$ by the input power of $300 \mathrm{~mW}$, the widths of short pulses generated at $2162 \mathrm{~nm}, 2141$ nm, $2122 \mathrm{~nm}$, and $2105 \mathrm{~nm}$ are compressed to be $85 \mathrm{fs}, 70 \mathrm{fs}, 55 \mathrm{fs}$, and 45 fs. 


\section{Conclusion}

In summary, we show that the red-shifted dispersive waves are efficiently generated from 2208 to $2105 \mathrm{~nm}$ by a section of PCF with a negative slope dispersion profile specially designed and fabricated in our lab. The conversion efficiency of $15 \%$ and wavelength-tunable range of over $100 \mathrm{~nm}$ are experimentally obtained. The high conversion efficiency and widely tunable mid-infrared spectrum can constitute an important progress in the development of a simple and compact ultrashort mid-infrared pulse source.

Acknowledgments This work is partly supported by the National Basic Research Program (2010CB327605 and 2010CB328300), the key grant of Ministry of Education (109015), the Program for New Century Excellent Talents in University (NECT-11-0596), Beijing Nova program (2011066), the Fundamental Research Funds for the Central Universities (2011RC008 and 2011RC0404), Open Fund of State Key Laboratory of Information Photonics and Optical Communications (Beijing University of Posts and Telecommunications) P. R. China, and Science Foundation Ireland (07/SK/I1200).

\section{References}

[1] J. M. Dudley, G. Genty, and S. Coen, Rev. Mod. Phys 78, 1135-1176 (2005).

[2] P. St. J. Russell, Science 299, 358-362 (2003).

[3] J. C. Knight, Nature 424, 847-851 (2003).

[4] A. V. Husakou and J. Herrmann, Phys. Rev. Lett. 87, 203901-203904 (2001).

[5] A. B. Fedotov, E. E. Serebryannikov, A. A. Ivanov, L. A. Mel'nikov, A. V. Shcherbakov, D. A. Sidorov-Biryukov, Ch.-K. Sun, M. V. Alfimov, A. M. Zheltikov, Laser Phys. Lett. 3, 301-305 (2006).

[6] V. P. Mitrokhin, A. A. Ivanov, A. B. Fedotov, M. V. Alfimov, K. V. Dukel'skii, A. V. Khokhlov, V. S. Shevandin, Yu. N. Kondrat'ev, A. A. Podshivalov, and A. M. Zheltikov, Laser Phys. Lett. 4, 529-533 (2007).

[7] K. S. Abedin, J. T. Gopinath, E. P. Ippen, C. E. Kerbage, R. S. Windeler, and B. J. Eggleton, Appl. Phys. Lett. 81, 1384-1386 (2002).

[8] D. A. Akimov, E. E. Serebryannikov, A. M. Zheltikov, M. Schmitt, R. Maksimenka, W. Kiefer, K. V. Dukel'skii, V. S. Shevandin, and Y. N. Kondrat'ev, Opt. Lett. 28, 1948-1950 (2003).

[9] S. O. Konorov, D. A. Akimov, A. A. Ivanov, M. V. Alfimov, A. B. Fedotov, D. A. Sidorov-Biryukov, L. A. Mel'nikov, A. V. Shcherbakov, I. Bugar, D. Chorvat Jr., F. Uherek, D. Chorvat, A. M. Zheltikov, Laser Phys. Lett. 1, 402-405 (2004).

[10] M. L. Hu, C. Y. Wang, L. Chai, Y. F. Li, K.V. Dukel'skii, A.V. Khokhlov, V. S. Shevandin, Yu.N. Kondrat'ev, and A.M. Zheltikov, Laser Phys. Lett. 1, 299-302 (2004).

[11] A. Kudlinski, V. Pureur, G. Bouwmans, and A. Mussot, Opt. Lett. 33, 2488-2490 (2008).

[12] J. H. Yuan, X. Z. Sang, C. X. Yu, S. G. Li, G. Y. Zhou, and L. T. Hou, IEEE J. Quantum. Electron. 46, 728-733 (2010).

[13] J. H. Yuan, X. Z. Sang, C. X. Yu, K. R. Wang, B. B. Yan, X. W. Shen, Y. Han, G. Y. Zhou, S. G. Li, and L. T. Hou, IEEE Photon. Technol. Lett. 24, 670-672 (2012).

[14] I. Cristiani, R. Tediosi, L. Tartara, and V. Degiorgio, Opt. Express 12, 124-135 (2004). 
[15] G. Q. Chang, L. J. Chen, and F. X. Kärtner, Opt. Lett. 35, 2361-2363 (2010).

[16] J. H. Yuan, X. Z. Sang, C. X. Yu, Y. Han, G. Y. Zhou, S. G. Li, and L. T. Hou, IEEE Photon. Technol. Lett. 23, 786-788 (2011).

[17] D. V. Skryabin, F. Luan, J. C. Knight, and P. St. J. Russell, Science 301, 1705-1708 (2003).

[18] B. W. Liu, M. L. Hu, X. H. Fang, Y. F. Li, L. Chai, C. Y. Wang, W. J. Tong, J. Luo, A. A. Voronin, and A. M. Zheltikov, Opt. Express 16, 14987-14996 (2008).

[19] H. H. Tu, and S. A. Boppart, Opt. Express 17, 9858-9872 (2009).

[20] M. Kolesik, L. Tartara, and J. V. Moloney, Phys. Rev. A 82, 045802-1-4 (2010).

[21] X. Z. Hu, L. S. Zou, and Y. X. Liu, Fiber Optic Cable and Engineering Application (Academic, Beijing, 1998).

[22] E. E. Serebryannikov, A. M. Zheltikov, S. Köhler, N. Ishii, C. Y. Teisset, T. Fuji, F. Krausz, and A. Baltuška, Phys. Rev. E 73, 066617-1-4 (2006).

[23] J. H. Peng, A. V. Sokolov, F. Benabid, F. Biancalana, P. S. Light, F. Couny, and P. J. Roberts, Phys. Rev. A 81, 031803(R)-1-4 (2010).

[24] N. Akhmediev, and M. Karlsson, Phys. Rev. A 51, 2602-2607 (1995). 